\title{
Clinical Microbiology Perspective of Empyema caused by Streptococcus constellatus in Malignancy Patient: A Case Report
}

\author{
Agung Dewi Sekar ${ }^{1}$, Rosantia Sarassari ${ }^{1 *}$, Sudarsono $^{2,3}$, Kuntaman $^{1,3}$ \\ ${ }^{1}$ Department of Medical Microbiology, Faculty of Medicine, Universitas Airlangga, Surabaya, Indonesia. \\ ${ }^{2}$ Department of Pulmonology and Respiratory Medicine, Faculty of Medicine, Universitas Airlangga, Surabaya, \\ Indonesia. \\ ${ }^{3}$ Dr. Soetomo General Hospital Surabaya, Indonesia.
}

\section{ARTICLE INFO}

Article history:

Received 7 February 2021

Received in revised form 27 May

2021

Accepted 28 May 2021

Available online 31 May 2021

\section{Keywords:}

Streptococcus constellatus,

Empyema,

Streptococcus anginosus group.

\begin{abstract}
Introduction: Streptococcus constellatus (SC) is commensal bacteria and belongs to Streptococcus anginosus group (SAG). However, SC causes infections especially in patient with underlying diseases. SC empyema is a clinical case that is described in very few studies, especially in Indonesia.

Case: A 45-year-old man was admitted to emergency department in Dr. Soetomo General Hospital Surabaya with respiratory insufficiency on November $11^{\text {th }} 2020$ after 3 months of non-productive cough and a week of weakness. He was diagnosed with empyema on right hemithorax and received chest tube insertion. SC was isolated from pleural fluid sample after first day of culture on Bactec BD bottle. GeneXpert result of pleural fluid was negative for Mycobacterium tuberculosis (MTB). He completed 10 days of intravenous ampicillin-sulbactam and metronidazole. The CT scan reported solid mass of $4.7 \times 7.4 \times 7.8 \mathrm{~cm}$ in posterolateral segment of inferior lobe right lung, right pleural effusion, with adenocarcinoma as biopsy result.

Discussion: SC is a normal commensal in respiratory tract, however with the presence of a certain factor such as immunocompromised, colonized SAG directly induces an infection after entering normal sterile sites in the body including pleural fluid.

Conclusion: Although infection caused by SC is a rare case, it still should be considered in clinical diagnosis and treatment of related infections, particularly in patients with comorbidities. The prognosis was good with appropriate antibiotics and chest tube insertion.
\end{abstract}

\section{INTRODUCTION}

Streptococcus constellatus (SC) is a grampositive, facultative anaerobic bacterium related to Streptococcus anginosus and Streptococcus intermedius. In general, these bacteria were included as Streptococcus anginosus group (SAG), formerly known as Streptococcus milleri group. They are commensal bacteria in respiratory, digestive, and reproductive tract. ${ }^{1}$ Nevertheless, recent evidence shows that they are also pathogenic and may cause infections. The incidence rate has reportedly increased continuously in the past two decades. $^{2}$ They are associated with abdominal, central nervous system, and pleuropulmonary infections. ${ }^{1,3} \mathrm{SC}$ empyema is a clinical entity that is described in the scientific literature in very few studies and with few patients, especially in Indonesia. This is a rare case report of Empyema caused by SC in patient with malignancy

*Correspondence: santisarassari@yahoo.com 


\section{CASE}

A 45 -year-old man with no underlying diseases who was admitted to the emergency room of public hospital with respiratory insufficiency on November $11^{\text {th }}$ 2020. The symptom was followed with general weakness. There was no history of loss of appetite, loss of weight, and night sweats two days before on November $9^{\text {th }} 2020$.

In the first three months, his symptoms began with non-productive cough, with no fever or other symptoms. Fifteen days before being admitted to Dr. Soetomo General Hospital, he went to a secondary referral hospital due to suffering pain in the chest and dyspnea. A total of $1,500 \mathrm{ml}$ of yellowish purulent fluid was removed. He was hospitalized for 3 days. After being discharged, he was referred to Puskesmas (primary health center/PHC) and was diagnosed as lung tuberculosis. He started consuming fixed dose combination of oral anti tuberculosis drugs (4FDC ATD) consisted of rifampicin, ethambutol, isoniazid, pyrazinamide for 10 days, but then he terminated the ATD due to vomitting.

Physical examination during the admission found asymmetrically thorax movement was decreasing on the right hemithorax. During palpation, fremitus was detected in half inferior of the right hemithorax, dullness in lower half of right hemithorax, pulmonary auscultation with diminished of respiratory sounds, and detected rhonchi on lower half of right hemithorax. The physical examination on November $13^{\text {th }} 2020$ revealed rhonchi was not detected on lower half of right hemithorax.

The infection marker results taken from complete blood tests on November $9^{\text {th }} 2020$ and November $12^{\text {nd }}$ 2020 were increased while on November $16^{\text {th }} 2020$ and November $19^{\text {th }} 2020$ were within normal range, listed in Table 1. Thorax chest X-ray showed parapneumonic effusion (PPE) on right lung (Figure 1). The CT scan reported solid mass of $4.7 \times 7.4 \times 7.8 \mathrm{~cm}$ in posterolateral segment of inferior lobe right lung, right pleural effusion. The fine needle biopsy of mass showed an adenocarcinoma.

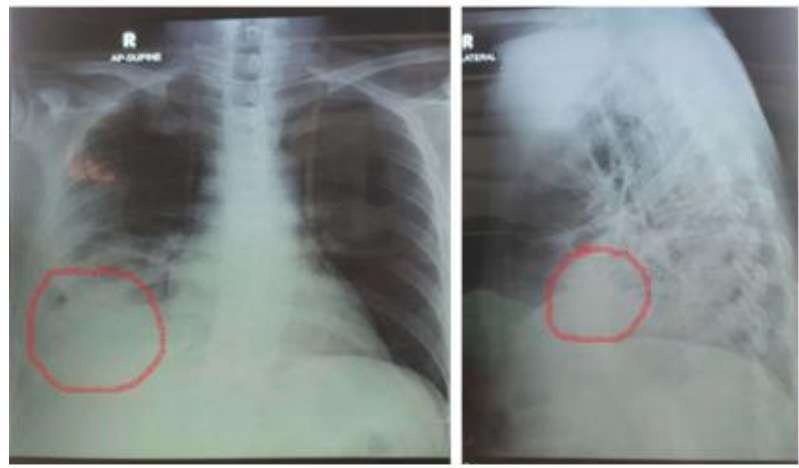

Figure 1. Chest X-Ray result from anteroposterior (A) and lateral (B). Showed parapneumonic effusion on right lung with chest tube inserted in right thoracic vertebrae 10 and 11

The microscopic examination revealed the acidfast stain was negative for acid fast bacilli and $\mathrm{KOH}$ staining was not identified as fungi or yeast. After 12 hours of incubation of blood sample in BD Bactec bottle, it showed positive result and then was subcultured into blood agar, chocolate agar, and MacConkey agar. One type of colony grew until $4^{\text {th }}$ quadrant on the blood agar and chocolate agar plate. The colony was small colonies, with alpha hemolytic. There was no bacterial growth on MacConkey agar. Gram stain of single colony was gram-positive cocci in chain. Bacterial ID and susceptibility result of pleural fluid using BD Phoenix ${ }^{\mathrm{TM}}$ was SC that was sensitive against linezolid, oxacillin, erythromycin, tetracycline, clindamycin, levofloxacin, vancomycin, chloramphenicol, penicillin, cotrimoxazole. There was no fungi growth on Sabouraud Dextrose Agar. The examination for Mycobacterium tuberculosis (MTB) with GeneXpert of pleural fluid was negative.

The yellowish fluid of about $50 \mathrm{ml}$ was removed using water seal drainage to relieve the shortness of breath. Codein pills and antibiotics were given to the patient. Ampicillin-sulbactam 1.5 gram every 6 hours and metronidazole $500 \mathrm{mg}$ every 8 hours were delivered to the patient for 10 days. After 10 days of antibiotics, the patient was discharged on November $20^{\text {th }}$ with no difficulty in breathing, no fever, no pain in chest tube insertion site, and no cough. 
Table 1. The results of blood infection markers

\begin{tabular}{|c|c|c|c|c|c|}
\hline & \multirow[t]{2}{*}{ Normal range } & \multicolumn{2}{|c|}{ Date } & \multirow[b]{2}{*}{$16 / 11 / 20$} & \multirow[b]{2}{*}{$19 / 11 / 20$} \\
\hline & & 9/11/20 & $12 / 11 / 20$ & & \\
\hline White Blood Cell & $3.37-10.0 \times 10^{3} \mu \mathrm{L}$ & $16.03 \times 10^{3} \mu \mathrm{L}$ & $\begin{array}{l}14.07 \mathrm{x} \\
10^{3} \mu \mathrm{L}\end{array}$ & $9.09 \times 10^{3} \mu \mathrm{L}$ & $9.94 \times 10^{3} \mu \mathrm{L}$ \\
\hline \multicolumn{6}{|l|}{ Diff count } \\
\hline Neutrophil & $39.8 \%-70.5 \%$ & $79,8 \%$ & $75.4 \%$ & $65.4 \%$ & $68.3 \%$ \\
\hline Lymphocyte & $13.1 \%-49.9 \%$ & $13,8 \%$ & $18.0 \%$ & $27.7 \%$ & $25.8 \%$ \\
\hline NLR* & $1-3$ & 5.78 & 4.18 & 2.37 & 2.65 \\
\hline Procalcitonin & $>0.5:$ systemic infection & - & 0.03 & 0.01 & 0.03 \\
\hline
\end{tabular}

Note: *NLR: Neutrophil- Lymphocyte ratio

\section{DISCUSSION}

$\mathrm{SC}$ is a gram-positive catalase-negative cocci. SC belongs to Streptococcus anginosus group (SAG) along with $S$. intermedius, and $S$. sanguis. SAG is commensal bacteria of digestive, respiratory, and reproductive tracts. However, SAG bacteria have been reported as known pathogen in the respiratory infections, ${ }^{1}$ which more often reported in patients with underlying disease ${ }^{2}$ than healthy patients. ${ }^{3}$ These bacteria can rarely cause pyogenic infections, such as abcess, empyema, arthritis, and intra-abdominal infections. ${ }^{4}$ Among pyogenic infections, empyema was third common manifestation after intra abdominal abscess and soft tissue abscess. ${ }^{5}$ The possible explanation of increased pathogenicity of these bacteria is cell surface adhesins, exotoxin production, evasion of phagocytosis, as well as synergism with anaerobic of polymicrobial infections. ${ }^{6}$ A molcular study detected multiple of pathogenicity factors in S. anginosus. ${ }^{7}$

Culture is a gold standard and most common technique used for isolating S. constellatus. However, the turn around time of culture usually takes 3-5 days. This would cause treatment delay. Multiplex PCR method for detection of six streptococci species has been developed. This technique gives rapid, simple, and reliable result for identification of these bacteria. ${ }^{8}$ Therefore, multiplex PCR can be used for identification and survaillance of these bacteria, along with culturebased method.

We reported a case in which SC caused an empyema in an immunocompromised patient. The initial presentation was non chronic non-productive cough increasing into shortness of breath with an empyema. The removal of purulent drainage and intravenous antibiotics improved the patient's symptoms.

Empyema is most commonly referred to pusfilled pockets which develop in the pleural space.
Empyema is a serious condition that requires treatment. $^{2,9,10}$ In this case, empyema in the patient happened as a complicated of PPE-confirmed on chest X-ray result. Complicated PPE was caused by pneumonia. Increasing passage of interstitial fluid as a result of inflammation associated with pneumonia along with bacterial invasion into pleural space leads to the increasing number of neutrophil and other inflammatory cells that cause complicated parapneumonic pleural effusion. The accumulation of pus in the pleural space causes an empyema. ${ }^{10,11}$

$\mathrm{SC}$ in the patient can be a causative agent for empyema. It is supported with the microbiology result which showed only one type of colony that grown from blood agar and chocolate agar. In addition, the laboratory results of infection marker such as leucocyte and neutrophil counts were also elevated.

Among SAG species, the infection caused by SC was $37.37 \%$ (173/463) with chest as the common site of infection after abdominal. The chest infections could have produced pulmonary abscess, pneumonia, pleural effusions, pyopneumothorax, and pleural fistula. The prevalence of pulmonary infection was higher in patients aged 55 years old and above. ${ }^{2}$ However, a case in a child was reported in $2020 .^{4}$ The preliminary results of this study found $6(1.14 \%)$ of 524 total pleural samples in 2015-2020 were SC.

Previous study showed colonized SAG directly induced noninvasive infections and caused invasive infections after entering normal sterile sites in the body including pleura. ${ }^{2}$ SAG can enter pleura through blood or lymph (hematogenous) or per continuitatem from lungs to pleura. $^{10}$ The underlying disease such as malignancy could cause a certain degree of immunosuppression which made SC easier to enter pleural space. Therefore, infection caused by SAG should be considered in clinical diagnosis and treatmentrelated infections. 
Generally, the prognosis of infection caused by $\mathrm{SC}$ is relatively less severe, sufficiently treated with simple drainage and intravenous antibiotics, had a shorter hospital stay, and lower than 30-day mortality rate compared to other SAG species. ${ }^{2,12}$

Those reports were similar to the patient in this case which clinically and laboratory improved after being hospitalized for 10 days and was treated with water seal drainage and intravenous combined antibiotics. Clinically, the patient's symptoms such as difficulty in breathing, cough, and weakness were decreasing after 2 days and were resolved after 10 days of therapy. This result was supported with physical examination that rhonchi on lower half of right hemithorax was not detected on $2^{\text {nd }}$ day of antibiotics. Furthermore, the blood infection markers were decreasing on the $1^{\text {st }}$ day of antibiotics (November $12^{\text {th }}$ 2020) and returned to normal range in $5^{\text {th }}$ day of antibiotics (November $16^{\text {th }} 2020$ ).

However, a report in the UK reported a patient with empyema caused by SC that required extensive decortication and prolonged hospital admission. ${ }^{12}$ Therefore, the pathogenic potential of commensal bacteria such as SCshould not be underestimated especially in patients with immunocompromised.

The antibiotic course is usually long, with mean duration of up to 34 days and most SAG isolates seem to be sensitive to penicillin and cefotaxime. ${ }^{13}$ Those data were similar to SC isolates in this case which are sensitive to penicillin. Some reports indicate that patients infected by SAG can be co-infected by other agents including an-aerobes. Thus, ampicillin-sulbactam and metronidazole were added at the beginning of the treatment.

\section{CONCLUSION}

As an opportunistic pathogen, SC was found to produce an empyema infection in patient with malignancy. Although it was a rare case infection caused by SC, it should be considered in clinical diagnosis and treatment of related infections After being treated with antibiotics and water seal drainage, the patient had a good outcom.

\section{REFERENCES}

1. Noguchi S, Yatera K, Kawanami T, et al. The Clinical Features of Respiratory Infections Caused by the Streptococcus anginosus Group. BMC Pulm Med 2015; 15: 133.

2. Jiang S, Li M, Fu T, et al. Clinical Characteristics of Infections Caused by Streptococcus anginosus Group. Sci Rep 2020; 10: 9032.

3. Alejandro MH, Laura PP. Empyema Caused by Streptococcus constellatus: Atypical Presentation of a Typical Pneumonia. J Clin Case Reports 2018; 8: $1-2$.

4. Vulisha AK, Sam R, Nur H, et al. Aggressive Presentation of Streptococcus constellatus. Cureus 2021; 13: e14534.

5. Kobo O, Nikola S, Geffen Y, et al. The Pyogenic Potential of the Different Streptococcus anginosus Group Bacterial Species: Retrospective Cohort Study. Epidemiol Infect 2017; 145: 3065-3069.

6. Parks T, Barrett L, Jones N. Invasive Streptococcal Disease: A Review for Clinicians. $\mathrm{Br}$ Med Bull 2015; 115: 77-89.

7. Asam D, Spellerberg B. Molecular Pathogenicity of Streptococcus anginosus. Mol Oral Microbiol 2014; 29: 145-155.

8. Hatrongjit R, Akeda Y, Hamada S, et al. Multiplex PCR for Identification of Six Clinically Relevant Streptococci. J Med Microbiol 2017; 66: 15901595.

9. Reis-Melo A, Soares D, Magalhães MF, et al. Complicated Pneumonia with Empyema Caused by Streptococcus Anginosus in a Child. Revista paulista de pediatria : orgao oficial da Sociedade de Pediatria de Sao Paulo 2020; 38: e2018258.

10. Kanai E, Matsutani N. Management of Empyema: A Comprehensive Review. Curr Challenges Thorac Surgery; Vol 2 (November 2020) Curr Challenges Thorac Surg, https://ccts.amegroups.com/article/view/37410 (2020).

11. Garvia V, Paul M. Empyema. Treasure Island (FL), 2021.

12. Chrastek D, Hickman S, Sitaranjan D, et al. Streptococcus constellatus Causing Empyema and Sepsis, Necessitating Early Surgical Decortication. Case Reports in Infectious Diseases 2020; 2020: 4630809.

13. Obszańska K, Kern-Zdanowicz I, Kozińska A, et al. Streptococcus anginosus (milleri) Group Strains Isolated in Poland (1996-2012) and Their Antibiotic Resistance Patterns. Polish J Microbiol 2016; 65: 33-41. 